Rev. Adm. Saúde Vol. 17, № 67, Abr. - Jun. 2017

http://dx.doi.org/10.23973/ras.67.28

ARTIGO DE REVISÃO

\title{
A análise da qualidade de medicamentos e o papel do laboratório oficial no contexto da saúde pública
}

Quality analysis of drug products and the role of the official laboratory in the context of public health

Márcia Lombardo ${ }^{1}$, Jaqueline Kalleian Eserian ${ }^{2}$

${ }^{1}$ Mestre em Fármaco e Medicamentos pela Faculdade de Ciências Farmacêuticas da Universidade de São Paulo, Pesquisador Científico do Centro de Medicamentos, Cosméticos e Saneantes, Instituto Adolfo Lutz, São Paulo, SP, Brasil.

${ }^{2}$ Mestre em Ciências pela Universidade Federal de São Paulo, Pesquisador Científico do Centro de Medicamentos, Cosméticos e Saneantes, Instituto Adolfo Lutz, São Paulo, SP, Brasil.

\section{RESUMO}

Os medicamentos são produtos de ampla utilização na sociedade, seja como medida curativa, paliativa ou profilática. As etapas para garantir a qualidade dos medicamentos são fundamentais ao sucesso terapêutico e devem se basear na ação eficaz e no uso seguro. Esta revisão bibliográfica teve como objetivo discutir a importância dos padrões de qualidade dos medicamentos na gestão de estabelecimentos de saúde, bem como na saúde dos pacientes que fazem uso destes produtos. Foram abordados assuntos relacionados às operações de controle, as quais devem ser executadas durante a fabricação e também o papel do laboratório oficial na avaliação da qualidade após a comercialização. A notificação de queixas técnicas e a realização de programas de monitoramento da qualidade de medicamentos oferecidos à população são ações de relevância no âmbito da saúde pública. O laboratório oficial atua como um mediador na investigação de ocorrências de desvios, de 
forma a contribuir com aspectos regulatórios e com a tomada de decisões por parte dos órgãos de vigilância sanitária.

Palavras-chave: Medicamento. Controle de qualidade. Farmacovigilância. Vigilância sanitária. Laboratório oficial. Análise fiscal de produtos.

\section{ABSTRACT}

Medicines are products widely used in society, either as a curative, palliative or prophylactic measure. The quality assurance of drug products is essential to the therapeutic success and should be based on effectiveness and safety. This review aimed to discuss the importance of the quality standards of drug products in the management of healthcare facilities, as well as for the health of the patients using this products. Issues related to quality control operations during the manufacture were addressed, as well as the role of the Official Laboratory in the evaluation of quality in the post-marketing period. The notification of technical complaints and the implementation of monitoring programs for the evaluation of drug products available to the population are actions of relevance to the public health. The Official Laboratory acts as a mediator in the investigation of deviations occurrences, in order to contribute to regulatory aspects and with the decision-making by the sanitary surveillance.

Keywords: Pharmaceutical preparations. Quality control. Pharmacovigilance. Health surveillance. Official laboratory. Fiscal analysis of products.

\section{INTRODUÇÃO}

Os medicamentos são considerados importantes instrumentos de saúde, seja para interromper o processo de adoecimento, diminuir o sofrimento ou melhorar a qualidade de vida do paciente. ${ }^{1}$ A Política Nacional de Medicamentos (PNM) aprovada no país em 1998 é um elemento da Política Nacional de Saúde que está fortemente relacionado à melhoria das condições da assistência à saúde. ${ }^{2}$

A PNM baseia-se nos princípios e diretrizes do Sistema Único de Saúde (SUS) e visa assegurar o acesso da população a medicamentos de qualidade, ao menor custo possível, nas três esferas de governo. Além disso, enfatiza a promoção do uso racional e a redução da automedicação, fortemente presente na sociedade brasileira. ${ }^{2}$

A organização da assistência farmacêutica sofreu mudanças significativas com a implantação da PNM, visto que alcançar uma atenção básica resolutiva dentro do SUS requer o acesso a medicamentos em momento oportuno, de maneira coerente, a produtos de qualidade e oferecendo todas as informações necessárias para o uso correto dos mesmos. ${ }^{3}$ Portanto, a assistência farmacêutica tornou-se um grande desafio, já que não só o 
medicamento é tido como elemento primordial mas também a sua relação com o usuário, em busca da efetivação de uma assistência terapêutica integral. ${ }^{4}$

A qualidade de medicamentos é um atributo de caráter não apenas comercial, mas também legal, ético e moral e o seu não cumprimento pode ocasionar sérias implicações à saúde pública. ${ }^{5} \mathrm{O}$ que se espera de um medicamento é que no momento do uso ele tenha ação farmacológica preservada e perfil toxicológico seguro. ${ }^{6}$

A garantia da qualidade de medicamentos é também uma importante ferramenta para a qualificação de fornecedores e o monitoramento do processo de compra no sistema público de saúde. A distribuição de medicamentos com desvio de qualidade pode colocar em risco a vida do paciente e onerar ainda mais o sistema, pois ao não se obter os resultados esperados o paciente entra em novo ciclo dentro do SUS, gerando novas consultas, exames e esquemas terapêuticos. ${ }^{7}$

Na gestão das unidades de saúde a administração de materiais se destaca como um ponto crítico. A oferta de produtos de qualidade, em quantidade e preço adequados são elementos chave na viabilização econômica. ${ }^{6} \mathrm{O}$ ideal é que a eleição dos fármacos para compor os esquemas terapêuticos seja feita segundo estudos de farmacoeconomia, os quais são baseados na relação custo-efetividade. ${ }^{8}$

A transição demográfica vivida no país está levando a uma demanda crescente dos recursos em saúde, sendo a aquisição de medicamentos apontada como uma das principais responsáveis pelo incremento nos gastos, especialmente no que se refere a medicamentos de alto custo, de uso prolongado ou para morbidades de elevada prevalência. Uma vez que os gastos estão aumentando progressivamente, as avaliações econômicas em saúde apoiadas em evidências científicas se mostram cada vez mais relevantes. ${ }^{9}$

A aquisição de medicamentos no setor público é baseada no binômio custo-qualidade. Medicamentos mais baratos mas com qualidade duvidosa podem gerar consequências negativas e maiores gastos. Além disso, o recolhimento de produtos sob suspeita de desvio de qualidade é um processo custoso e que compete com outras demandas prioritárias do sistema. $^{7}$

Os atributos de qualidade a serem considerados durante o processo de aquisição podem estar relacionados não só a questões intrínsecas do medicamento, tal como a eficácia, como também à sua adequabilidade ao perfil nosológico do serviço de saúde, o grau de exigência pretendido e o que pode ser feito efetivamente para garantir esta exigência. ${ }^{6}$

A aquisição de medicamentos de baixa qualidade muitas vezes pode ser atribuída a motivos como: cadastro desatualizado de empresas fabricantes e distribuidoras, baixo índice de notificação compulsória de desvios de qualidade, ausência de comprovação do registro do medicamento, regulamentação insuficiente e inexistência de um sistema de informação ao consumidor. ${ }^{10}$

A licitação na modalidade menor preço gera preocupação com a qualidade dos produtos distribuídos à população. No entanto, existem mecanismos para o adequado julgamento das propostas, como a inserção de critérios de habilitação do licitante, a aptidão no desempenho das atividades e a idoneidade técnica. Além de assegurar a eficácia e segurança dos 
produtos, o monitoramento da qualidade de medicamentos integrado a ações de farmacovigilância contribui com o aprimoramento do processo licitatório. ${ }^{6,7}$

O objetivo deste trabalho foi discutir a importância dos parâmetros de qualidade na produção de medicamentos, abrangendo conceitos relacionados ao controle de qualidade durante a fabricação, bem como a avaliação da qualidade como parte das ações da vigilância sanitária, seja em casos de suspeitas e queixas técnicas ou por meio de programas rotineiros no período pós-comercialização, destacando a atuação do laboratório oficial neste processo.

\section{METODOLOGIA}

A pesquisa da literatura foi realizada por meio de busca on-line nas bases de dados Scielo e Google Acadêmico, utilizando as palavras-chave: controle de qualidade de medicamentos, queixa técnica de medicamentos, vigilância sanitária e laboratório oficial. Os artigos foram selecionados de acordo com a abordagem direta do tema e a disponibilidade eletrônica, resultando na escolha de 27 artigos, publicados entre 1999 e 2016, nas modalidades original, revisão, nota técnica, relato de experiência e editorial. Para complementar o tema foram também consultadas outras fontes como capítulos de livros e endereços eletrônicos de órgãos oficiais contendo guias, informações técnicas e material jurídico.

\section{RESULTADOS E DISCUSSÃO}

O laboratório do Ministério da Saúde ou congênere da união, dos estados, do distrito federal e dos territórios que apresenta competência delegada, através de convênio ou credenciamento, para realizar a análise de drogas, medicamentos, insumos farmacêuticos e correlatos é denominado laboratório oficial. ${ }^{11} \mathrm{~A}$ avaliação da qualidade de produtos envolvidos em queixas e denúncias, bem como a participação em programas periódicos para o monitoramento de produtos comercializados ou distribuídos à população são as atividades preponderantes do laboratório oficial. A relevância destas atividades será discutida com maior profundidade nos tópicos apresentados a seguir.

\section{Controle versus avaliação da qualidade de medicamentos}

Para alcançar o objetivo da qualidade de maneira confiável é fundamental que o fabricante do medicamento implemente um sistema de garantia de qualidade, o qual deve incorporar todos os instrumentos legais pertinentes e os requisitos mínimos que possibilitem o cumprimento destes instrumentos. ${ }^{12,13}$

As normas de Boas Práticas de Fabricação e Controle de medicamentos (BPFC) regulamentadas pela Agência Nacional de Vigilância Sanitária pretendem assegurar a produção consistente e controlada, utilizando padrões de qualidade apropriados para o uso requerido no registro do produto. ${ }^{14}$ 
O cumprimento das BPFC está dirigido à diminuição dos riscos inerentes à produção, ou seja, contaminação cruzada, contaminação por partículas, troca de produtos ou mistura de produtos. Compete ao controle de qualidade realizar operações capazes de verificar a conformidade do produto com as especificações de qualidade, compreendendo etapas de amostragem, execução de ensaios analíticos, julgamento dos resultados e documentação. Ressalta-se que o controle de qualidade não deve se limitar a operações laboratoriais de análise e sim estar envolvido em todas as decisões relacionadas à qualidade do produto. ${ }^{14,15}$

As atividades de validação dos métodos de análise são prioritárias para o cumprimento das práticas de controle, permitindo o desenvolvimento de métodos eficazes e com níveis apropriados de exatidão, precisão e confiabilidade. ${ }^{12} \mathrm{~A}$ necessidade de se mostrar a qualidade de medições é cada vez mais reconhecida e exigida, já que dados analíticos inadequados podem ter consequências desastrosas e prejuízos financeiros irreparáveis. ${ }^{16} \mathrm{O}$ processo de validação de métodos se caracteriza por estudos experimentais voltados ao atendimento das exigências das aplicações analíticas, assegurando resultados confiáveis durante o uso rotineiro. ${ }^{17}$

As matérias-primas utilizadas na produção farmacêutica estão sujeitas à análise e cumprimento de requisitos de qualidade, a fim de garantir a máxima segurança do produto final. Além dos testes habituais de liberação, o teste de identificação da matéria-prima deve ser realizado para todos os recipientes de todos os lotes. ${ }^{13}$

A avaliação da estabilidade da formulação farmacêutica é importante para prever características específicas do processo produtivo, a possibilidade de interação entre os componentes, os fatores ambientais a que pode ser submetida e o material de embalagem mais adequado para seu acondicionamento. ${ }^{6}$

A estabilidade de formulações farmacêuticas se expressa pela sua integridade, de maneira a não comprometer a liberação e a absorção do ativo. A perda da estabilidade físico-química pode acarretar em degradação de componentes, modificação na concentração do ativo e/ou formação de produtos potencialmente tóxicos. A perda da estabilidade microbiológica pode significar ineficácia do sistema de conservação ou do processo esterilizante, bem como a contaminação em níveis acima do permitido. ${ }^{6}$

Enquanto o controle de qualidade realizado pelo fabricante do medicamento constitui a sua filosofia de produção, o laboratório oficial é responsável pela avaliação da qualidade mínima do produto disponível ao usuário. Portanto, não é função do laboratório oficial garantir a qualidade de qualquer linha de produção ou realizar controle de qualidade, embora as técnicas e métodos utilizados sejam praticamente os mesmos. ${ }^{18}$

Tal avaliação reflete a percepção estatal do risco à saúde e se materializa em normas jurídicas, a serem observadas pelo setor fabril e fiscalizadas pela vigilância sanitária. Do ponto de vista da saúde pública, a menção dos motivos da apreensão do produto a ser avaliado é fundamental para orientar e diferenciar a abordagem laboratorial. Desse modo, o laboratório oficial deverá dar primazia aos testes capazes de elucidar o problema em questão. $^{18}$

A ocorrência de desvios de qualidade de medicamentos pode ser devida a falhas humanas ou falhas em processos de manufatura, transporte ou armazenamento. ${ }^{19} \mathrm{~A}$ não-conformidade 
resultante representa um somatório de atribulações para o fabricante, principalmente prejuízos de re-trabalho, perda de credibilidade e cassação da licença de funcionamento e de registro. Para o paciente, a falta de qualidade pode trazer sérias complicações à saúde. ${ }^{15}$

Cabe à vigilância sanitária promover ações voltadas à avaliação da qualidade de produtos farmacêuticos disponíveis no mercado na ocorrência de suspeita ou denúncia de adulteração, falsificação, alteração em características físico-quimicas ou falhas terapêuticas. ${ }^{15}$

Segundo a literatura, existem duas categorias de medicamentos de baixa qualidade, 0 contrafeito e o de padrão inferior. O primeiro refere-se a erros deliberados ou fraudes e 0 segundo, a desvios de qualidade sem dolo proposital. ${ }^{20}$

O medicamento contrafeito pode abranger produtos de referência, similares ou genéricos com alteração em sua identidade e/ou origem, principalmente a ausência ou insuficiência de componentes ativos ou mesmo o uso de outros componentes ativos no lugar dos usuais. Tais produtos não apresentam qualquer benefício terapêutico e podem ser responsáveis por causar resistência e até mortes. ${ }^{21}$

A falsificação de produtos com fins terapêuticos ou medicinais constitui infrações sanitárias e crimes contra a saúde pública, citados no Código Penal. O consumo destes medicamentos pode causar enormes prejuízos, sendo indispensável a ampliação das estratégias de fiscalização e de pesquisa laboratorial, bem como de ações educativas por parte das agências regulatórias, órgãos policiais e governo federal. ${ }^{22}$

A apreensão de produtos apontados em denúncias feitas por usuários ou profissionais de saúde pretende acionar as autoridades sanitárias para apuração e tomada de providências, resgatando o caráter investigativo das ações de vigilância. Produtos com alteração ou adulteração evidente requerem respostas rápidas e a legislação determina a obrigatoriedade de interdição cautelar sempre que houver indícios de perigo iminente para a saúde. ${ }^{18}$

Os laboratórios oficiais são partes fundamentais no processo de elucidação das ocorrências, contribuindo com as ações de proteção à saúde pública e oferecendo subsídios para a regulação no âmbito sanitário. ${ }^{23}$ Afinal, retirar produtos do mercado que são carentes de eficácia e segurança é também contribuir com a promoção do uso racional de medicamentos. ${ }^{24}$

\section{A queixa técnica de medicamentos}

Os estudos farmacoepidemiológicos fornecem informações a respeito do perfil de utilização de medicamentos em uma população, evidenciando as suas consequências sanitárias, sociais e econômicas, bem como os aspectos associados à gestão saúde/doença. ${ }^{8}$

A situação dos países em desenvolvimento merece atenção particular em relação à segurança do paciente no uso de medicamentos, pois com frequência estes países enfrentam dificuldades no abastecimento, na aquisição de produtos de qualidade e na habilitação do profissional de saúde, tornando maior a probabilidade de ocorrência de eventos adversos a medicamentos. ${ }^{25}$ 
O campo da farmacovigilância dedica-se a identificar, avaliar e prevenir os riscos ou qualquer outro problema relacionado a medicamentos, tais como reações adversas, ineficácia, dependência, abuso, intoxicação, erros de medicação e desvios de qualidade. ${ }^{26}$

Os erros de prescrição, dispensação e administração, a dificuldade de adesão e o uso de medicamentos impróprios, manipulados incorretamente ou com integridade comprometida podem ser considerados erros de medicação. ${ }^{27}$ Os desvios de qualidade em medicamentos se caracterizam por alterações no produto, seja em propriedades organolépticas, parâmetros fisico-químicos, parâmetros biológicos ou informações técnicas de bulas e rótulos. ${ }^{28}$

As crescentes inovações tecnológicas em produtos farmacêuticos e para a saúde têm demonstrado a necessidade de fortalecer a vigilância pós-uso/pós-comercialização (VIGIPÓS). A capacitação de uma rede sentinela e de um sistema de notificação é necessária para que estes atuem como interface das informações relacionadas a eventos adversos e queixas técnicas de medicamentos. ${ }^{23}$ Os incidentes relacionados ao uso de medicamentos podem ser circunstâncias notificáveis como potencial evento adverso (near miss), evento adverso sem dano, evento adverso com dano ou queixa técnica. ${ }^{29}$

A queixa técnica de medicamentos ocorre quando se observa um afastamento dos parâmetros de qualidade pré-estabelecidos para o produto, muitas vezes em consequência a práticas ilegais. A notificação de queixa técnica é avaliada para a classificar o risco sanitário e planejar a estratégia de ação, desencadeando ações de inspeção, coleta, análise fiscal ou adoção de medidas preventivas. ${ }^{28}$

À semelhança dos medicamentos, os produtos para saúde tão presentes no ambiente de hospitais e clínicas, tais como equipamentos, materiais, artigos médicos, implantes e itens para diagnóstico também são alvo da vigilância sanitária. Problemas na qualidade destes produtos devem ser notificados e enquadrados no escopo da tecnovigilância. ${ }^{19}$

Alguns trabalhos demonstraram que as queixas mais recorrentes em notificações envolvendo medicamentos e produtos para saúde realizadas por hospitais de diferentes regiões do país foram atribuídas a problemas na embalagem, como vazamento e dificuldade de abertura; alteração no aspecto do produto, como cor e integridade, falhas na quantidade total disponível na embalagem e erros em informações do rótulo. De um modo geral, estas queixas foram caracterizadas pela fácil visualização do problema antes mesmo da utilização do produto. ${ }^{19,29,30}$

Segundo Caon, Feiden e Santos (2012), a maior parte dos trabalhos sobre farmacovigilância apresentam ênfase na descrição de efeitos adversos sem indicar possíveis ocorrências na qualidade do medicamento, sendo que esta abordagem também merece atenção especial, pois um medicamento com suspeita de desvio de qualidade pode ser a causa de uma reação adversa. $^{30}$

A eficácia terapêutica de medicamentos depende de múltiplos fatores, dentre eles a qualidade do produto, o uso correto e o estado clínico do paciente. Portanto, é importante que a investigação de ineficácia terapêutica envolva a análise laboratorial do produto, principalmente diante de casos graves. ${ }^{31,32}$ Os ensaios analíticos relacionados com a eficácia, de um modo geral, se restringem à identificação e à quantificação das substâncias ativas declaradas no registro. ${ }^{18}$ 
As notificações de suspeitas de desvio de qualidade de medicamentos representam um avanço no processo de regulação sanitária, pois não só auxiliam a melhoria da qualidade do produto como possibilitam estabelecer o perfil das ocorrências, e com isto, qualificar a assistência. $^{30}$

Tendo em vista a carência de informações científicas neste tópico, a atuação das Instituições Sentinela é fundamental. A alimentação dos sistemas de notificação contribui com a avaliação da realidade das queixas técnicas no país e com o norteamento da elaboração de novos critérios de controle. ${ }^{30} \mathrm{~A}$ partir das informações obtidas poderão ser realizados alertas de segurança, atualização da legislação ou recomendações sanitárias. ${ }^{23}$

O exercício dos profissionais de saúde no relato de queixas técnicas deve ser aprimorado por meio de estratégias educativas que demonstrem a importância dessa atitude para a segurança do paciente. ${ }^{19}$ É imprescindível que a notificação seja orientada e incentivada por todos da equipe. ${ }^{29}$

\section{O monitoramento da qualidade de medicamentos}

As apreensões planejadas de amostras de medicamentos pela vigilância sanitária configuram os programas de fiscalização, cujo objetivo é verificar elementos que o fiscal não pode constatar a olho nu, tais como a identidade e teor de fármaco. Estes programas devem ser detalhados quanto às metas e critérios de eleição dos produtos, tendo a participação do laboratório oficial na definição do plano de amostragem. ${ }^{18}$

O monitoramento da qualidade de medicamentos visa anteceder possíveis riscos e danos aos pacientes, sendo a divulgação das informações obtidas úteis na qualificação de fornecedores de interesse ao SUS. ${ }^{7}$ A construção de programas de monitoramento pactuados entre os entes do Sistema Nacional de Vigilância Sanitária tem o objetivo de verificar a qualidade dos produtos utilizados no âmbito nacional. ${ }^{23}$

Os indicadores gerados podem ser aplicados no processo de seleção, aquisição e utilização, assim como no subsídio de possíveis intervenções dos órgãos regulamentadores. ${ }^{19}$ É a partir do indicador de qualidade que o gestor será capaz de traçar medidas de controle e também realizar ações de educação continuada, a fim de minimizar a ocorrência de falhas relacionadas ao medicamento. ${ }^{33}$

A avaliação periódica da qualidade dos medicamentos disponibilizados no SUS deve incluir produtos que já foram notificados com suspeita de desvio de qualidade, produtos que apresentaram laudo anterior insatisfatório e também aqueles produtos que são os mais consumidos no mercado, criando-se um modelo de intervenção preventiva. O monitoramento tem como foco avaliar parâmetros de identidade, pureza, eficácia, inocuidade e integridade. ${ }^{34}$ Os resultados satisfatórios obtidos nestes programas indicam o cumprimento de requisitos que viabilizam um tratamento eficaz. ${ }^{35,36}$

Bianchin et al. (2012) afirmam que existem poucos trabalhos científicos divulgando os resultados do monitoramento da qualidade de medicamentos fornecidos pelo SUS e atentam para elevados índices de reprovação de produtos com teor de fármaco abaixo do esperado 
ou próximo do limite de aceitação, relacionando tal desvio com o uso de matérias-primas de má qualidade, a ocorrência de degradação ou o uso proposital de limites inferiores com intuito de reduzir custos. ${ }^{7}$

De acordo com Silva (2000) um fabricante idôneo não admite a liberação de um lote com teor próximo a um dos limites extremos, tendo em vista que este limite deverá manter-se estável até o último dia do prazo de validade. O fabricante, dispondo de todos os dados da produção, pode elevar ao máximo os padrões de controle, visando a obtenção de lotes homogêneos. ${ }^{18}$

No âmbito institucional interno os programas de monitoramento da qualidade de medicamentos favorecem as atividades de educação permanente, com o intuito de garantir uma maior segurança ao paciente. No âmbito institucional externo eles fornecem informações qualificadas para tomada de decisão dos órgãos de vigilância sanitária do país. ${ }^{29}$ E importante que estes programas estejam articulados à PNM e a uma lógica que considere a morbimortalidade da população, incluindo os medicamentos prioritários e que respondam às necessidades de saúde. A implementação, a continuidade e o aprimoramento dos programas de monitoramento são um grande desafio para a área da saúde pública. ${ }^{1}$

\section{A atuação do laboratório oficial}

De acordo com a Constituição Federal, a vigilância sanitária é preconizada como um dos campos de atividade do SUS e seus componentes tradicionais incluem a fiscalização, a inspeção, a regulação e o monitoramento. ${ }^{37,38} \mathrm{~A}$ vigilância sanitária a que ficam sujeitos os medicamentos e insumos farmacêuticos é instituída pela lei 6.360/1976, a qual trata essencialmente de normas para o registro, a autorização de funcionamento, a responsabilidade técnica, o controle de qualidade, a rotulagem, a publicidade, os meios de transporte, as infrações e as penalidades. ${ }^{39}$

A velocidade de introdução de novos produtos e tecnologias supera os conhecimentos acumulados e consolidados em atos sanitários, fazendo com que novas fontes de agravo à saúde precisem ser monitoradas com base em regulamentações específicas. A elaboração das mesmas somente é possível se houver interação entre o agente identificador do agravo e o laboratório oficial, que buscará elucidar e comprovar as causas por meio de análises previstas na legislação e que servirão como complemento à ação de fiscalização. ${ }^{18}$

Desse modo, os laboratórios oficiais são instituições relevantes na geração do conhecimento a ser sistematizado e disseminado, auxiliando epidemiologicamente a redução ou a eliminação do risco potencial, ou seja, do risco sanitário. ${ }^{38}$

O Sistema Nacional de Laboratórios de Saúde Pública (SISLAB) é formado por um conjunto de redes nacionais de laboratórios, organizadas em sub-redes, por agravos ou programas, de forma hierarquizada. Suas ações abrangem as esferas federal, estadual e municipal, em conformidade com os princípios do SUS. Os laboratórios centrais (Lacens) estão vinculados às secretarias estaduais de saúde e têm como uma de suas competências a avaliação da qualidade analítica das redes estaduais. ${ }^{23}$ 
Os Lacens produzem informações que fortalecem o Sistema Nacional de Vigilância Sanitária, resultantes de investigações de desvios de qualidade, de atendimento a rotinas e de elaboração de programas e projetos de avaliação de produtos e serviços. Assim, acredita-se que os Lacens também podem ser notificadores de grande importância no controle de agravos à saúde da população. ${ }^{23}$

As modalidades de análises previstas em lei estão relacionadas ao momento do ciclo da fiscalização, apresentando objetivos específicos e diferentes graus de implicação para a saúde pública. A modalidade análise prévia é aquela que ocorre durante o processo de concessão do registro, enquanto a modalidade análise de controle visa a avaliação da capacidade de produzir segundo os termos concedidos no registro, verificando se o produto que está entrando na cadeia de consumo corresponde aos aspectos previamente aprovados. $^{18}$

A modalidade análise fiscal é a que mais vem sendo praticada e avalia a capacidade do fabricante em seguir produzindo medicamentos com qualidade satisfatória durante toda a vida útil do produto. O termo de apreensão de amostra representa a lavratura do auto de fiscalização e o elo de ligação entre a inspeção e a avaliação analítica, favorecendo a relação de causalidade entre os fatos apurados e o evento identificado. Assim, a descrição minuciosa das suspeitas deve acompanhar as amostras apreendidas e orientar a abordagem analítica. $^{18}$

A análise macroscópica é um método que exige pouco ou nenhum equipamento e permite uma primeira avaliação, de forma não destrutiva. Apesar de alguma inferência sobre a qualidade a partir de características macroscópicas ou organolépticas, ela não permite qualquer determinação mais apurada sobre a estabilidade ou esterilidade das formas farmacêuticas. Assim, deve-se arbitrar até que ponto pretende-se exigir a inclusão de procedimentos que privilegiem a identificação, teor de ativo e uniformidade de doses, dentre outros testes. ${ }^{6}$

A análise laboratorial seguirá normas que frequentemente consideram limites de tolerância em seus critérios de aceitação. A monografia oficial é específica para o produto e aplica-se a qualquer tamanho amostral, seguindo o princípio de que qualquer unidade do produto disponível no mercado deve estar dentro dos limites de tolerância estabelecidos. ${ }^{18}$

O laudo analítico mostra se o produto preenche ou não as características estabelecidas na legislação sanitária, devendo informar a necessidade ou não de intervenção na fonte de agravo. Trata-se de uma prova científica que só pode ser contestada com evidências equivalentes, sendo a perícia de contraprova um desdobramento do laudo condenatório. A análise de desempate é determinada por um novo exame pericial, a ser realizado na segunda amostra, em poder do laboratório oficial. Os laudos que classificam o produto como impróprio para consumo desencadeiam processos de inspeção, sendo que qualquer delonga desnecessária à superação da fonte de agravo poderá trazer danos incalculáveis à saúde dos usuários. ${ }^{18}$

As infrações à legislação sanitária federal estão configuradas na lei $6.437 / 1977 .{ }^{40}$ Esta lei estabelece as bases do sistema processual sanitário e realça a correlação entre constituição e processo. O feito sanitário tem como padrão a medida punitiva, porém, revela-se como um instrumento essencial na investigação do nexo causal entre a conduta do autuado e o evento 
que deu causa à infração. A decisão administrativa confere efetividade à atuação estatal e a via processual compatibiliza a garantia dos direitos do autuado com o bem intangível, que é a saúde pública. ${ }^{41}$

\section{CONSIDERAÇÕES FINAIS}

Como subsídio às ações de proteção à saúde realizadas pela vigilância sanitária, cabe ao laboratório oficial elucidar problemas relacionados à qualidade de produtos disponíveis no mercado, por meio de análises físicas, químicas, biológicas ou técnicas. No caso dos medicamentos, a garantia do binômio segurança e eficácia merece atenção especial, visto que estes produtos são utilizados com frequência por uma população que se encontra vulnerável e que impreterivelmente necessita de uma resolução efetiva dos agravos em sua saúde.

\section{REFERÊNCIAS}

1. Junior DMP, et al. A definição de medicamentos prioritários para o monitoramento da qualidade laboratorial no Brasil: articulação entre a vigilância sanitária e a Política Nacional de Medicamentos. Cad Saúde Pública. 2008; 24(9): 2081-2090.

2. Brasil. Secretaria de Políticas de Saúde. Política Nacional de Medicamentos. Portaria $n$. 3.916/GM, 30 outubro de 1998. Diário Oficial da União no.15-E, Seção I, pág. 18 a 22, 10 novembro de 1998.

3. Oliveira LCF, Assis MMA, Barboni AR. Assistência Farmacêutica no Sistema Único de Saúde: da Política Nacional de Medicamentos à Atenção Básica à Saúde. Ciênc Saúde Coletiva. 2010;15(Suppl 3): 3561-3567.

4. Tavares N, Pinheiro R. Assistência Farmacêutica no SUS: avanços e desafios para a efetivação da assistência terapêutica integral. Rev Tempus Actas Saúde Col. 2014;8(1):4956. doi:10.18569/tempus.v8i1.1452.

5. Gil ES. Controle físico-químico de qualidade de medicamentos. 3a.ed. São Paulo: Pharmabooks; 2010.

6. Luiza VL, Castro CGSO, Nunes JM. Aquisição de medicamentos no setor público: o binômio qualidade-custo. Cad Saúde Pública. 1999;15(4):769-776.

7. Bianchin MD, et al. Avaliação da qualidade de comprimidos de propanolol e enalapril distribuídos no sistema público de saúde em uma cidade do sul do Brasil. Ciênc Saúde Coletiva. 2012;17(2):491-498.

8. Cunha MCN, Zorzatto JR, Castro LLC. Avaliação do uso de medicamentos na Rede Pública Municipal de Saúde de Campo Grande/MS. Rev Bras Ciênc Farm. 2002;38(2):215227. 
9. Faria ACM, et al. Estudos farmacoeconômicos no Brasil: onde estamos? Rev Bras Farm Hosp Serv Saúde. 2014;5(4):13-18.

10. Ivama MA, Noronha $A B$, Hofmeister MGS. Prevenção e combate à falsificação e fraude de medicamentos: uma responsabilidade compartilhada. Brasília: Organização PanAmericana da Saúde/Organização Mundial da Saúde; 2005 [citado 6 fev. 2017]. Disponível em:

http://www.paho.org/bra/index.php?option=com_docman\&task=doc_view\&gid=802\&ltemid=4 23.

11. Brasil. Casa Civil. Dispõe sobre o Controle Sanitário do Comércio de Drogas, Medicamentos, Insumos Farmacêuticos e Correlatos, e dá outras Providências. Lei n. 5.991, 17 dezembro de 1973. Diário Oficial da União, Seção I, pág. 13049, 19 dezembro de 1973.

12. La Roca MF, et al. Desenvolvimento e validação de método analítico: passo importante na produção de medicamentos. Rev Bras Farm. 2007;88(4):177-180.

13. Amorim SR, Klier AH, Angelis LH. Controle de qualidade na indústria farmacêutica: identificação de substâncias por espectroscopia no infravermelho. Rev Bras Farm. 2013;94(3):234-242.

14. Brasil. Agência Nacional de vigilância sanitária. Boas Práticas de Fabricação de Medicamentos. Resolução RDC no.17, 16 abril de 2010. Diário Oficial da União, Seção I, pág. 94-110, 19 abril de 2010.

15. Peixoto MM, et al. Avaliação da qualidade de comprimidos de captopril dispensados em Feira de Santana-BA. Infarma. 2005;16(13-14):69-73.

16. Ribani M, et al. Validação em métodos cromatográficos e eletroforéticos. Quim Nova. 2004;27(5):771-780.

17. Brasil. Agência Nacional de vigilância sanitária. Guia para validação de métodos analíticos e bioanalíticos. Resolução RE no. 899, 29 de maio de 2003. Diário Oficial da União, Seção I, pág. 56-59, 2 jun de 2003.

18. Silva ACP. O laboratório oficial na avaliação analítica. In: Rozenfeld S. Fundamentos da vigilância sanitária. 6a.ed. Rio de Janeiro: Fiocruz; 2000. p. 271-301.

19. Azulino ACO, et al. Queixas técnicas realizadas pelos profissionais da saúde, relacionadas aos produtos utilizados em Hospital Sentinela de Belém-Pará. Rev Bras Farm Hosp Serv Saúde. 2013;4(3):13-16.

20. Newton PN, Green MD, Fernández FM. Impact of poor-quality medicines in the 'developing' world. Trends Pharmacol Sci. 2010;31(3-3):99-101. doi:10.1016/j.tips.2009.11.005.

21. World Health Organization. Counterfeit medicines: the silent epidemic. Geneva; 2006 [citado 8 fev. 2017]. Disponível em: http://www.who.int/mediacentre/news/releases/2006/pr09/en/. 
22. Ames J, Souza DZ. Falsificação de medicamentos no Brasil. Rev Saúde Pública. 2012;46(1):154-159.

23. Branco NMC, et al. NOTIVISA e os Laboratórios de Saúde Pública: A interface da informação em vigilância sanitária. Vigil Sanit Debate. 2015;3(3):130-134. doi:10.3395/2317269x.00242.

24. Aquino DS. Por que o uso racional de medicamentos deve ser uma prioridade? Ciênc Saúde Coletiva. 2008;13(Suppl):733-736.

25. World Health Organization. Quality of care: patient safety. Geneva; 2002 [citado em 8 fev 2017]. Disponível em: http://www.who.int/patientsafety/worldalliance/ea5513.pdf.

26. Centro de vigilância sanitária. O que é farmacovigilância? cvs.saude.sp.gov.br [Internet]. São Paulo: Secretaria de Estado da Saúde; [citado em 8 fev. 2017]. Disponível em: http://www.cvs.saude.sp.gov.br/apresentacao.asp?te_codigo=22

27. Cassiani SHB. A segurança do paciente e o paradoxo no uso de medicamentos. Rev Bras Enferm. 2005;58(1):95-99.

28. Sistema Nacional de Notificações para a Vigilância Sanitária. Formulário para Notificação de Queixa Técnica de Medicamento. Brasília: Agência Nacional de vigilância sanitária [citado 8 fev. 2017]. Disponível em:

http://www.anvisa.gov.br/hotsite/notivisa/manual/qt_medicamento.pdf.

29. Lima PF, et al. Queixas técnicas e eventos adversos a medicamentos notificados em um hospital sentinela do interior de São Paulo, 2009-2010. Epidemiol Serv Saúde.

2013;22(4):679-686. doi:10.5123/S1679-49742013000400014

30. Caon S, Feiden IR, Santos MA. Desvios de qualidade de medicamentos em ambiente hospitalar: identificação e avaliação das ocorrências. Rev Bras Farm Hosp Serv Saúde. 2012;3(1):23-26.

31. Lombardo M, Eserian JK. Investigação da suspeita de desvio de qualidade em uma solução injetável de citrato de fentanila. Infarma. 2015;27(3):191-194. doi:10.14450/23189312.v27.e3.a2015.pp191-194.

32. Lombardo M, Eserian JK. O controle da hemorragia pós-parto e a avaliação da qualidade da ocitocina injetável. Perspect Médicas. 2016;27(1): 26-31.

doi:10.6006/perspectmed.20160103.5115322278

33. Bezerra ALQ, et al. Análise de Queixas Técnicas e Eventos Adversos Notificados em um Hospital Sentinela. Rev Enferm. 2009;17(4):467-472.

34. VISA Goiás. Monitoramento de medicamentos. Goiânia: vigilância sanitária de Goiás [citado 8 fev. 2017]. Disponível em:

http://www.visa.goias.gov.br/post/ver/128171/monitoramento-de-medicamentos. 
35. Eserian JK, Lombardo M. Monitoramento da qualidade de comprimidos de clonazepam distribuídos na rede pública estadual de São Paulo e a sua contribuição para o sucesso terapêutico. Rev Eletronica Farm. 2015;XII(4): 57-64.

36. Eserian JK, Lombardo M. Avaliação da qualidade de comprimidos revestidos de diclofenaco de sódio dispensados pela rede pública municipal de São Paulo, SP, Brasil. Infarma. 2016;28(3):185-190. doi:10.14450/2318-9312.v28.e3.a2016.pp185-190.

37. Sandri MMS, De Seta MH, Luiza VL. Autoridades reguladoras de medicamentos sulamericanas: uma análise a partir de regras organizacionais. Rev Panam Salud Publica. 2013; 34(3):169-175.

38. Gemal AL, Araújo DG, Delgado IF. Laboratório de vigilância sanitária: segurança sanitária na história da saúde pública. Vigil Sanit Debate. 2015;3(3):1-3. doi:10.3395/2317269x.00632.

39. Brasil. Congresso Nacional. Dispõe sobre a vigilância sanitária a que ficam sujeitos os Medicamentos, as Drogas, os Insumos Farmacêuticos e Correlatos, Cosméticos, Saneantes e Outros Produtos, e dá outras Providências. Lei n. 6.360, 23 setembro de 1976. Diário Oficial da União, Seção I, pág. 12647, 24 setembro de 1976.

40. Brasil. Congresso Nacional. Configura infrações à legislação sanitária federal e estabelece as sanções respectivas. Lei n. 6.437, 20 agosto de 1977. Diário Oficial da União, Seção I, pág. 11145, 24 agosto de 1977.

41. Delgado JS. O processo administrativo-sanitário como instrumento de efetividade das ações de vigilância sanitária. Rev Tempus Actas Saúde Col. 2013;7(1):225-240. doi: 10.18569/tempus.v7i1.1290.

Recebido: 12 maio 2017. Publicado: 29 maio 2017

Correspondência: Márcia Lombardo. Instituto Adolfo Lutz, Centro de Medicamentos, Cosméticos e Saneantes. Avenida Doutor Arnaldo, 355, Prédio BQ, 50 andar, CEP 01246902, São Paulo, SP. E-mail: mlombardo@ial.sp.gov.br

Conflito de Interesses: os autores não declararam conflito de interesses

(C) This is an Open Access article distributed under the terms of the Creative Commons Attribution License, which permits unrestricted use, distribution, and reproduction in any medium, provided the original work is properly cited. 